\title{
Transport sédimentaire de la dune à la zone du déferlement sur une plage sableuse soumise à des vents de terre
}

\author{
François Sabatier $^{(\mathrm{a}, \mathrm{b})}$, Olivier Samat
(b)
Adrien Lambert
}

(a)

Delft University of Technology, Faculty of Civil Engineering, Hydraulic Engineering Section, Stevinweg 1, 2628 CN Delft, The Netherlands. sabatier@cerege.fr,

CEREGE - Centre Européen de Recherche et d'Enseignement en Géosciences de l'Environnement, Europôle de L'Arbois, BP 80, 13545 Aix-en-Provence cedex04,

(c) France.lambert@cerege.fr, samat@cerege.fr

Département de Géographie, Université de Safi, Maroc. chaibi1@caramail.fr

CETE Méditerranée, B.P. 37000, 13791 Aix-en-Provence Cedex3,

France.Frederic.Pons@equipement.gouv.fr

\section{Résumé}

Des mesures instantanées par pièges à sables depuis la dune jusqu’à la zone du déferlement et la comparaison de 10 profils sur une plage sableuse microtidale du Golfe du Lion pendant des épisodes de vents de terre et d'accrétion du rivage ont été conduites. La berme se déplace vers la mer et son érosion par le vent participe à l'engraissement de la zone du jet de rive. Les volumes déplacés dans la partie sous marine sont 6 à 100 fois supérieurs à ceux mis en mouvement sur la plage.

\section{Abstract}

Field measurements (sediment traps and profile survey) during off-shore wind period on a sandy beach demonstrate aeolien sediment transport from the beach to the beach face. Gross sediment transport on the nearshore zone is more important than gross sediment transport on the beach by a factor 6 to 100 .

\section{Introduction}

Sur les plages sableuses, les variations morphologiques et le transport sédimentaire sont étudiés depuis près d'un siècle maintenant $\mathbf{1}$. Des progrès considérables ont été réalisés dans la description des états de plages en fonction de l'hydrodynamique , dans la définition et la quantification du transit littoral dans la zone du déferlement et dans l'évolution des dunes sous l'effet du vent . L'ensemble des travaux antérieurs a permis de décrire la morphodynamique des plages, de réaliser des modèles conceptuels et d'étalonner des formules de transport en vue d'une approche modélisatrice . Aujourd'hui cependant, la majeure partie des recherches continue à étudier la zone littorale par unités morphologiques distinctes : dune, berme, zone du jet de rive, barres d'avant côte. Très peu de travaux expérimentaux prennent en compte les processus de manière intégrée sur l'ensemble du profil de plage depuis la dune à la zone du déferlement. 
De plus, si les processus durant les tempêtes sont majoritairement étudiés ${ }^{6}$, les mécanismes d'accrétion en relation avec les houles de beau temps demeurent encore un champ de recherche à développer car leur compréhension permettra une description morphodynamique de la plage de l'échelle événementielle à saisonnière et annuelle . Pour apporter des éléments novateurs sur la morphodynamique des plages sableuses en période d'accrétion associée à des vents de terre et à des houles de faible énergie, nous présentons ici des mesures par pièges à sables et une analyse de profils sur le site de Rousty (Camargue, Golfe du Lion) (fig.1). Les récents travaux français sur la dynamique éolienne des plages d'Atlantique , de la Manche et de la Mer du Nord , ont mis en évidence les connections du système dune/plage mais dans des contextes différents du site présenté ici. Ces côtes sont surtout soumises à des vent de mer, au contraire du littoral du Golfe du Lion où l'importance des vents de terre ne peut être négligée (Mistral et Tramontane). Le profil étudié (D50 environ de 0,2 mm) comporte une dune, une berme et deux barres parallèles au rivage et correspond au type « Dissipative-Bar-Trough » .

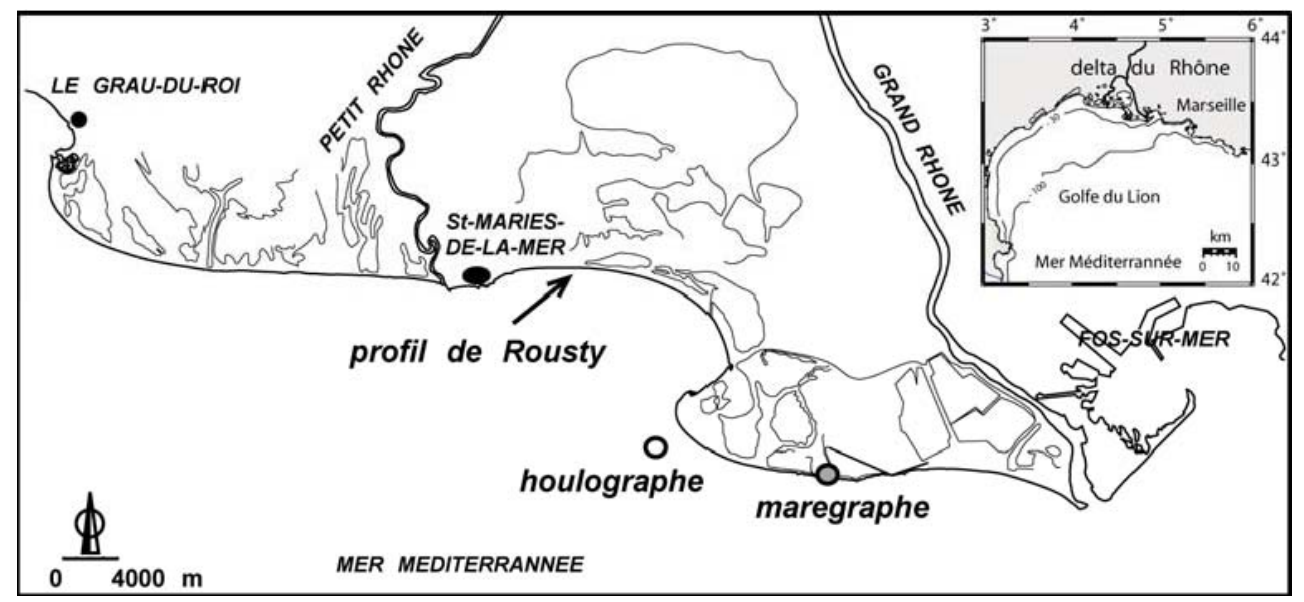

Figure.1. Localisation du site d'étude

\section{Méthodes}

\subsection{Pièges à sables de la dune à la zone du déferlement}

Des mesures par pièges à sables conventionnels ont été conduites sur la plage émergée et dans la zone du déferlement le 26 février 2001. Sur la plage émergée, nous avons utilisé 8 pièges de type «Leatherman » déployés sur toutes les unités morphologiques de la plage, depuis la dune jusqu'à $6 \mathrm{~m}$ de la limite supérieure du jet de rive. Les pièges de 0,75 $\mathrm{m}$ de haut sont constitués de tubes PVC enfoncés verticalement dans le sable (fig.2). Dans la zone du déferlement, quatre pièges de type "Kraus " ont été déployés simultanément aux pièges de la plage émergée. Chaque piège est constitué d'une structure accueillant 2 à 6 nacelles réparties verticalement qui piègent les sables dans le sens du courant (fig.2). Ces structures sont maintenues manuellement dans la zone du déferlement (le transport du jet de rive n'est pas pris en compte). Les pièges ont été déployés simultanément pendant une durée de 20 minutes pour la plage émergée (" Leatherman ») et durant 4 minutes dans la zone du déferlement ("Kraus »). Les pièges « 
Leatherman » permettent de prendre en compte le transport éolien qui est unidirectionnel pendant les coups de vents. En revanche, des essais d'utilisation des pièges "Kraus » pour capter le transport cross-shore se sont révélés infructueux à cause des filets qui s'emmêlaient autour des structures nous imposant de ne considérer que le transport longshore (dominant pendant nos mesures du fait de l'obliquité de la houle déferlante par rapport au rivage). On reprochera aux pièges de perturber les écoulements de l'air ou de l'eau et de générer des affouillements à leur base, mais dans la gamme des types de pièges existants, ces modèles offrent le meilleur compromis efficacité/mesures
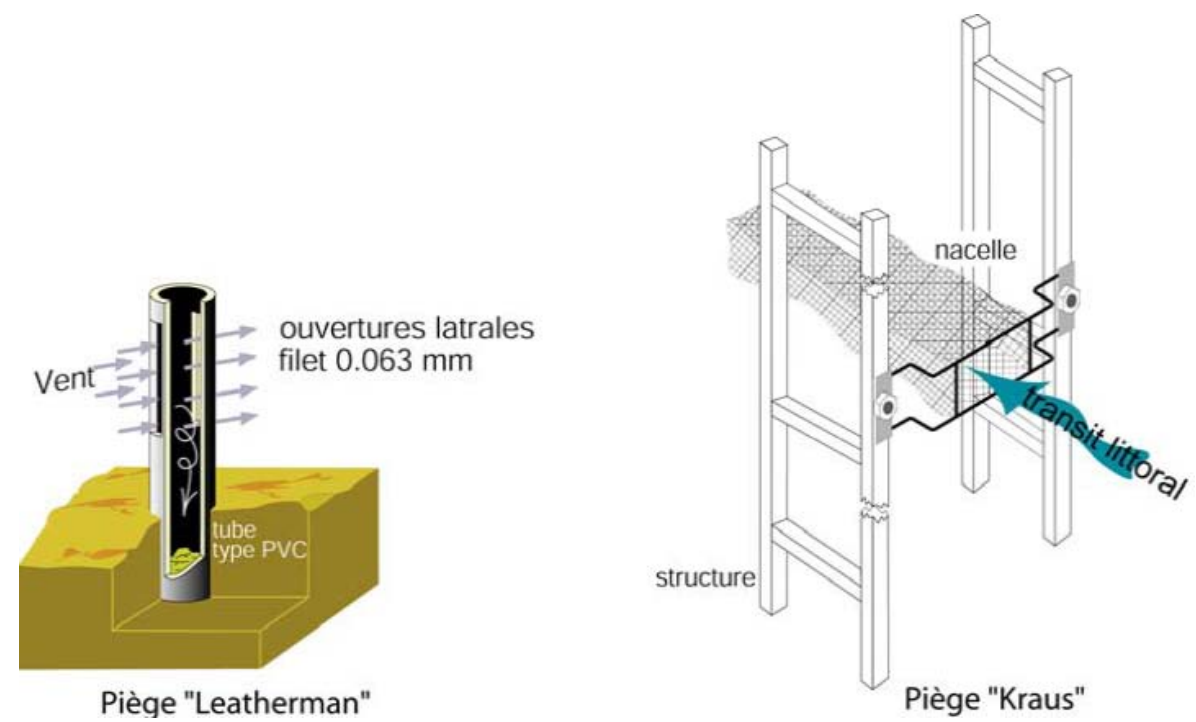

Figure.2. Pièges à sables utilisés

\subsection{Relevés morphologiques du profil}

Des relevés topographiques et bathymétriques ont été réalisés au moyen d'un tachéomètre et d'un échosondeur couplé à un DGPS embarqué sur un bateau pneumatique. La surveillance morphologique du profil de Rousty a duré une année (2000-2001) selon une périodicité de relevés toutes les deux semaines environ. De cette base de mesures, nous avons extrait 10 relevés répondant à quatre critères afin de ne conserver que les profils contrôlés par le vent de terre et les houles de beau temps: (1) dominance des vents de terre, (2) conditions de faible agitation (Hmo $<0,8 \mathrm{~m})$, (3) niveau de la mer bas n'inondant pas la plage ( $<+0,5 \mathrm{~m} \mathrm{NGF)} \mathrm{et} \mathrm{(4)} \mathrm{accrétion} \mathrm{de}$ la face à la mer de la berme

\subsection{Mesures de forçages}

Le 26 février 2001, le vent a été mesuré au moyen d’une station météo Davis à 0,75m du sol, positionnée sur la crête de la berme avec une acquisition de force et direction moyennes toutes les minutes. Dans la zone du déferlement, la hauteur des vagues déferlantes est estimée au mât à houle. La station Météo-France aux Saintes-Maries-de-la-Mer et le marégraphe des Salins du Midi situés respectivement à 2 et $10 \mathrm{~km}$ du site sont utilisés pour caractériser le vent et les variations du plan d'eau durant le suivi morphologique (fig.1). 


\section{Résultats et discussion}

\subsection{Transport sédimentaire de la dune à la zone du déferlement}

Lors de l'expérimentation du 26 Février 2001, le vent soufflait entre 6 et 9 m.s ${ }^{-1}$, légèrement 11

au dessus du seuil de mise en mouvement des sables . La houle au large est haute (Hmo) de 1,0 $\mathrm{m}$ avec un période (Tp) de 5,3 secondes tandis qu'au déferlement, elle atteint 0,38 $\mathrm{m}$ associée à un angle de 12,5 degrés environ induisant un courant de dérive littorale de $0,22 \mathrm{~m} . \mathrm{s}^{-1}$. Ces conditions sont particulières car elles doivent permettre l'utilisation des pièges à sables dans les deux milieux. Lorsque la mer est plus agitée, l'utilisation des pièges "Kraus " n'est pas réalisable techniquement, tandis que les pièges "Leatherman » seraient utilisables sur la plage. L'expérimentation in situ par pièges à sables n'est donc envisageable que dans des conditions de faible énergie.

Le transport éolien cross-shore varie le long du profil de plage en fonction de la morphologie (fig.3). Depuis l'arrière dune au sommet de celle ci le transport augmente puis diminue ensuite jusqu'au pied de la dune. A partir de cette zone, le transport s'accroît ensuite jusqu'au sommet de la berme et diminue encore avant le jet de rive. Le transit littoral longshore (fig.3) est relativement homogène sur les pièges positionnés sur la barre interne et dans la fosse car la houle ne déferlait pas de manière régulière sur la barre durant l'expérimentation. Le transit est maximal tout près du rivage, au dernier point de déferlement, avant le jet de rive, en relation avec un déferlement permanent à ce niveau. Les sables mis en suspension par le déferlement sont ensuite transportés parallèlement au rivage par le courant de dérive littorale. En terme de volumes transportés, le transit littoral est largement supérieur au transport éolien. Les valeurs, au niveau des pièges varient de 20 à 1,5 kg.min.m dans la partie sous marine contre 0,008 à 0,3 kg.min.m sur la plage émergée. Sur l'axe du profil, le transport total est estimé à 0,36 kg.min sur la plage et à 42 kg.min par la dérive littorale. Le transit littoral est donc environ 100 fois supérieur au transit éolien sous les forçages mesurés pendant l’expérimentation.

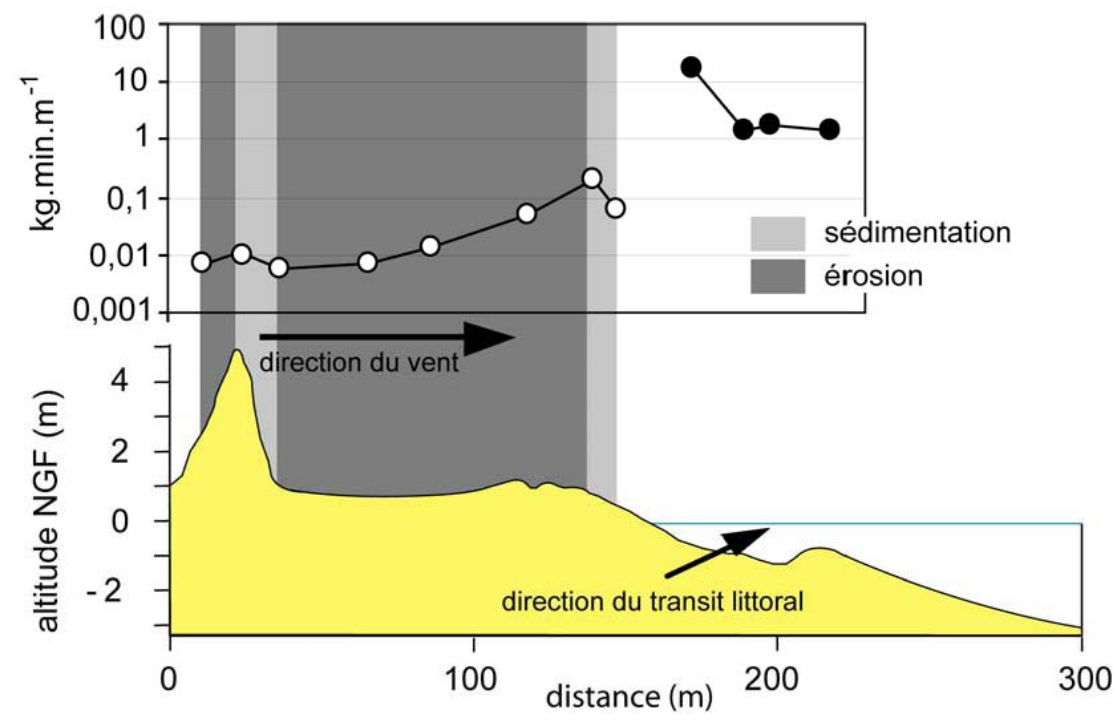

Figure.3. Transport sédimentaire éolien (blanc) et longshore (noir) interprété comme un modèle conceptuel de fonctionnement pendant un épisode de vent de terre 
En utilisant les formules classiques de transport sédimentaire de Bagnold ${ }^{4}$ et de Komar et 3 14, 15 Inman , validées et calibrées à partir des mêmes pièges à sables ${ }_{3}^{14}$, le transport éolien annuel représente $11 \mathrm{~m}$.m contre $70.000 \mathrm{~m}$.m pour le transit littoral (le transport sédimentaire crossshore de la plage immergée n'est pas pris en compte). En se basant sur les calculs théoriques, même si cette méthode demeure relativement imprécise, le transit littoral est donc environ 6000 fois supérieur au transport éolien. Ces ordres de grandeur sont largement supérieurs à ceux obtenus avec les mesures instantanées par pièges à sables. La différence provient probablement des formules théoriques qui considèrent le transport sédimentaire à l'équilibre.

\subsection{Processus d'accrétion de la berme}

Les variations du transport dans le profil sont interprétées en terme de bilan sédimentaire décrivant le fonctionnement de la plage émergée pendant les épisodes de vent de terre (Mistral) ${ }^{16}$. Cette approche n'est pas réalisable pour la partie sous-marine car le transport mesuré est parallèle au rivage et le gradiant cross-shore du transport n'est donc pas connu. Le bilan sédimentaire de la plage est négatif par vents de terre, dans le cas mesuré, de $0,056 \mathrm{~kg} . \mathrm{min}^{-1} \mathrm{~m}^{-1}$. Ces valeurs sont relativement faibles car elles correspondent à des vitesses de vents légèrement supérieures à la limite du transport éolien. Le fait le plus important concerne l'augmentation du transport depuis le pied de la dune jusqu'au sommet de la berme qui indique une érosion de cette zone (0,2 kg.min.m ${ }^{-1}$ ) (fig.3). Les sables sont dirigés vers la mer et sédimentent sur la face à la mer de la berme $\left(0,15\right.$ kg.min.m $\left.{ }^{-1}\right)$ très probablement en raison d'une baisse de la vitesse du vent liée à la morphologie de la plage. Les observations de terrain indiquent que le transport éolien s'effectue jusqu'à la zone du jet de rive où les sables sont ensuite repris par la dynamique marine. La zone du jet de rive est donc partiellement alimentée par le transport éolien.

La part des apports éoliens dans l'accrétion du rivage est estimée à partir des 10 profils de plage présélectionnés (cf 2.2) en déduisant de l'accrétion de la face à la mer de la berme l'érosion de la face à la dune de la berme (fig.4). La participation des sables transportés par le vent de terre à l'accrétion de la face à la mer de la berme est de $45 \%$ en moyenne mais cette valeur masque d'importantes variations (écart type $=21$ ) où deux situations extrêmes sont possibles. La première, durant laquelle les apports éoliens sont extrêmement réduits et participent pour $10 \%$ seulement à l'accrétion de la face à la mer de la berme. La seconde où l'accrétion de la face à la mer de la berme est causée par 80 \% d’apports éoliens. L’avancée du rivage durant les périodes de beau temps, liée à l'accrétion de la berme est donc principalement causée par des apports sableux de la partie sous-marine souvent associée à un déplacement des barres . Cependant la part des apports éoliens ne doit pas être négligée lorsque l’on quantifie les échanges sédimentaires dans le profil. Ce processus explique probablement les difficultés à modéliser l'accrétion à partir des outils classiques d’ingénierie qui ne prennent pas en compte le transport éolien. 


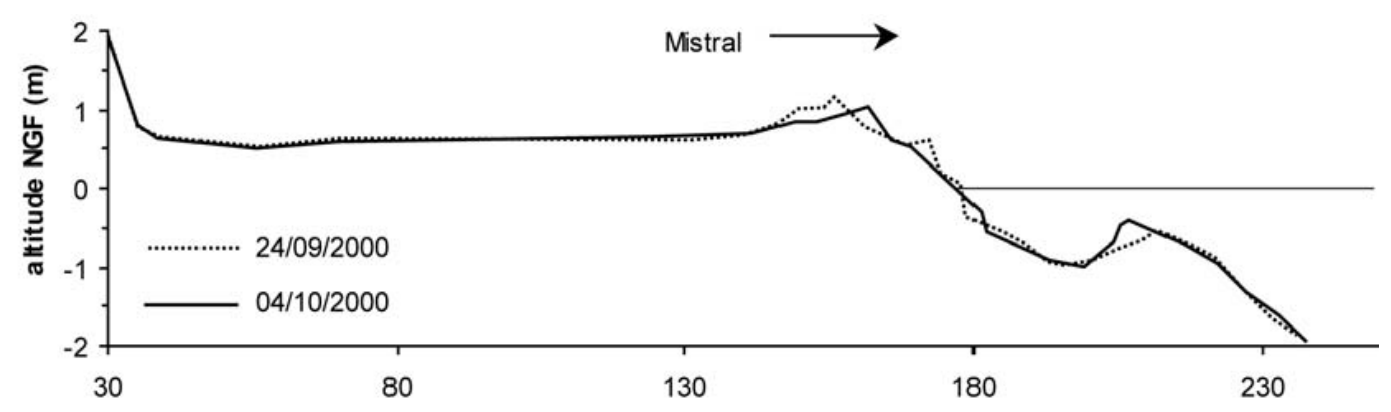

Figure.4. Déplacement de la berme vers la mer sous l'effet d'un vent de terre associée à un déplacement de la barre interne vers le rivage.

\section{Conclusion}

La morphodynamique des plages soumises à des vents de terre accompagnée d'une accrétion du rivage demeure encore peu étudiée. En terme de transport instantané, le rapport entre le transport éolien et le transit littoral varie d'un facteur 100 d'après les mesures par pièges à sables et 6000 à partir de calculs théoriques. Les mesures in-situ (pièges et profils) révèlent l'importance de la zone de la face à la mer de la berme qui s'engraisse par les apports éoliens et la dynamique marine durant les épisodes de houles de beau temps. Ce concept peut probablement être généralisé pour d’autres plages du Golfe du Lion aux forçages similaires (vent de terre Tramontane- et accrétion durant la période estivale). Cependant, l'expérimentation dans la zone du jet de rive proprement dite par pièges à sables n'a pu être conduite et les travaux futurs devront quantifier les transits dans cette zone pour mieux en comprendre la morphodynamique.

Nos résultats présentent finalement une application concrète dans le cas de rechargements artificiels en sables de la plage émergée. En effet nous mettons en évidence les capacités naturelles du vent à redistribuer les sables vers le rivage. Des dépôts artificiels de sables sur la plage seraient donc cohérents avec les dynamiques naturelles.

\section{Remerciements}

Ce travail fait parti du programme de recherche LITEAU « Conception d'outils de gestion des plages sableuses » financé par le MATE. André Hérault (CEREGE) a contribué à la réalisation des pièges à sables. La commune des Saintes-Maries-de-la-Mer est remerciée pour avoir permis l'accès en véhicule au site de mesures. Le Conseil Général des Bouches-du-Rhône, la Région PACA et la Compagnie des Salins du Midi et des Salines de l'Est sont remerciés pour leur aide financière apportée à ce travail.

\section{Références}

1 Johnson J., (1919). Beach Erosion Board. U.S. Army, 432 p

2 Wright, L.D., Short, A.D. and Green, M.O., (1985). Short-term changes in the morphodynamics states of beaches and surf zones : an empirical predictive model. Marine Geology, 62, 339-364.

3 Komar, P.D. and Inman, D.L., (1970). Longshore sand transport on beaches. Journal of Geophysical Research, 75, (30), 5914-5927.

4 Bagnold, R.A., (1941). The physics of blown sand and desert dunes, 265 p.

5 Stive M.J.F. et Reniers A.J.H., (2003). Sandbars in motion. Science, 299, 18551856. 
6 Lee, G.-H., Nicholls, R.J. and Birkemeier, W.A., (1998). Storm-driven variability of the beach-nearshore profile at Duck, North Carolina, USA, 19811991. Marine Geology, 148, 163177.

7 Hoefel F. et Elgar S., (2003) Wave-induced sediment transport and sandbar migration, Science, 299, 1885-1887.

8 Pedreros R., (2000). Quantification et modélisation du transport éolien au niveau des zones côtières. Application au littoral Girondin. Thèse, Université de Bordeaux 1, 196 p.

9 Bretel P. (2003). Processus éoliens et morphodynamqiue dunaire sur les côtes bas normandes. Thèse, Université de Caen, 312 pages

10 Vanhee S. (2002). Processus de transport éolien à l'interface plage macrotidale-dune bordière : le cas des plages à barres intertidales, côte d'Opale, Nord de la France. Thèse, Université du Littoral Côte d'Opale, 233 p.

11 Leatherman, S.P., (1978). A new aeolian sand trap design. Sedimentology, 25, 303-306.

12 Kraus, N.C., (1987). Application of portable traps for obtaining point measurements of sediment transport rates in the surf zone. Journal of Coastal Research, 3, (2), 139-152.

13 Rosati, J. and Kraus, C., (1988). Hydraulic test of streamer sediment trap.Journal of Hydraulic Enginerring, 114, 12, 1527-1532.

14 Sabatier F. (2001). Fonctionnement et dynamiques du littoral du delta du Rhône. Thèse, Université Aix-Marseille III, 272 p.

15 Sabatier F., Chaibi M., et Pons F., (2002). Validation of aeolian sediment transport formulae, in E. Özhan (Ed.), International MEDCOAST Workshop on Beaches of the Mediterranean and the Black Sea: Dynamics, Regeneration, Ecology \& Management, Turkey, 87-98.

16 Nordstrom, K.F. Bauer, B.O., Davidson-Arnott, R.G.D., Gares, P.A., Carter, R.G.W., Jackson, D.WT., Sherman, D.J., (1996). Offshore aeolian transport across a beach : Carrinck Finn Strand, Ireland. Journal of Coastal Research, 12 (3): 664-672.

17 Larson M. et Kraus N. C. (1994). Temporal and spatial scales of beach profile change, Duck, North Carolina. Marine Geology, 117, (1-4), 75-94.

18 Sabatier F., Lambert A., Chaibi M., Samat O., Provansal M. (2002) Morphodynamique du profil de plage en milieu microtidal : du relevé de terrain au modèle mathématique et numérique. Geomorphology : from expert opinion to modelling, 26-27 avril 2002, 204-208. 